\title{
Decreased miR-503 expression in gastric cancer is inversely correlated with serum carcinoembryonic antigen and acts as a potential prognostic and diagnostic biomarker
}

\author{
This article was published in the following Dove Press journal: \\ OncoTargets and Therapy \\ 23 December 2016 \\ Number of times this article has been viewed
}

\author{
Daoyi Wu \\ Gaojian Cao \\ Zhenfeng Huang \\ Kai Jin \\ Haowei Hu \\ Jie Yu \\ Yu Zeng
}

Department of Gastrointestinal Surgery, The Third Affiliated Hospital of Wenzhou Medical University, Ruian, People's Republic of China
Correspondence: Yu Zeng Department of Gastrointestinal Surgery, The Third Affiliated Hospital of Wenzhou Medical University, 108 Wansong Road, Ruian, Zhejiang 325200, People's Republic of China Email562988116@qq.com

\begin{abstract}
Background: Altered expression of miR-503 has been linked to human carcinogenesis. In this present study, we aimed to detect the potential for miR-503 as a novel biomarker for gastric cancer (GC) patients.
\end{abstract}

Materials and methods: The relative mRNA level of miR-503 in serum and tissue of $68 \mathrm{GC}$ patients and serum of 32 healthy volunteers was determined by real-time reverse transcription quantitative polymerase chain reaction.

Results: The miR-503 level was significantly lower in the tissue and serum of GC than their counterparts (all $P<0.01$ ). Downregulation of miR-503 was found to be corrected with more aggressive tumor. Patients in the high-miR-503 group showed significantly better overall survival compared to the low-miR-503 group $(P=0.021)$. The serum miR-503 level in GC was inversely correlated with carcinoembryonic antigen $(\mathrm{CEA})(r=-0.624, P<0.001)$. Furthermore, the area under the receiver operating characteristic curve for miR-503 discriminating GC patients from healthy individuals was $0.889(P=0.006)$, with a sensitivity of $96.8 \%$ and a specificity of $79.4 \%$, higher than that of CEA (area under the receiver operating characteristic curve $=0.681, P=0.048$ ).

Conclusion: The present study suggests that the expression level of miR-503 may serve as prognostic and diagnostic biomarker for GC.

Keywords: microRNA, gastric cancer, diagnosis, prognosis, biomarker

\section{Introduction}

Gastric cancer (GC) is the second leading cause of cancer-related deaths in People's Republic of China. According to the data of 2012, there were about 951,600 new cases and 723,100 deaths. ${ }^{1}$ Surgical resection remains the main treatment for GC, despite advances in early diagnosis and improvements in therapeutic regimen. Majority of GC are present at advanced stage with either metastatic lesion or extensive local/ regional disease, and less than 30\% patients among those can undergo curative resection, with a very low 5 -year overall survival (OS) rate $(<10 \%)$. However, earlystage GC has an extremely good chance of being cured by endoscopic resection or less invasive surgical regimen; the 5-year survival for early-stage GC after curative resection can reach up to $100 \%{ }^{2}$ Thus, promising noninvasive biomarkers for identifying high-risk individuals, in combination with the current main diagnostic technology, scheduled follow-up endoscopy, are still urgently needed.

MicroRNAs (miRNAs), a class of small regulatory ( 25-28 nucleotides), noncoding RNAs, have emerged as important regulators by involving in various 
physiological and pathological events of human tumor entitles, such as cellular differentiation, apoptosis, growth, and metastasis in carcinogenesis and deterioration. ${ }^{3,4}$ The miRNA profile correlates tightly with the development and prognosis of human tumors, and cumulative evidence has confirmed that the expression levels of miRNAs may be indicators of the intrinsic characteristics of tumors. In addition, miRNAs can be reliably detected and quantified in common diagnostic biological materials such as fine-needle aspiration biopsy samples and formalin-fixed paraffin-embedded samples, even minimally invasive materials such as peripheral blood, serum, body fluid, and sputum, and are practical for assessment by standard biotechnology approaches. ${ }^{5}$ Taken together, miRNAs have been identified as a kind of new innovative biomarkers for improving cancer patients' prognosis, diagnosis, and treatment. ${ }^{6,7}$

MiR-503 has been identified to downregulate in GC specimens and cell lines. ${ }^{8}$ In addition, it has been proved as a key modulator of cell biological behaviors in GC: miR-503 inhibits GC cell malignant behaviors and inhibits epithelial-mesenchymal transition (EMT) in GC cells. ${ }^{8}$ By targeting IGF1R and BCL2, miR-503 sensitizes human GC cell lines to cisplatin of human GC cell lines. ${ }^{9}$ However, a little is known about the association between the expression level of miR-503 and clinicopathological parameters and prognosis in GC, and the expression of miR-503 has not yet been reported in the sera of GC patients.

In the present study, we aimed to detect the expression of miR-503 in tissue and serum samples of GC patients and healthy individuals, to investigate the association between its expression levels and clinicopathological parameters, and to determine whether miR-503 could be a novel biomarker for GC.

\section{Materials and methods}

\section{Human tissue samples and clinical data collection}

A total of 68 patients underwent resection of the primary GC at The Third Affiliated Hospital of Wenzhou Medical University and were enrolled in this study. The histological diagnosis of GC was confirmed by two pathologists, through the evaluation of hematoxylin and eosin-stained tissue sections. No patient received preoperative chemotherapy or radiotherapy. Resected tissue samples were immediately immersed in liquid nitrogen and stored at $-80^{\circ} \mathrm{C}$ for further processing. Detailed information regarding age, sex, OS, and GC features such as tumor size, histological grade, depth of invasion, status of lymphatic metastasis, and distant metastasis were collected retrospectively form all the patients (Table 1). Clinical stage of GC was evaluated on the basis of the criteria of the WHO Classification of Tumours of the Digestive System, 2010 edition. ${ }^{10}$ Patient follow-up was performed until April 1, 2016. The patients who died from other diseases or unexpected events were excluded from this study; during follow-up, 43 patients died of GC.

\section{Serum collection}

Serum samples were obtained from 32 GC patients (randomly selected from the aforementioned patients for tissue sample collection). Additionally, 32 healthy individuals with no history of cancer or other diseases were recruited. All samples were collected from the patients' antecubital vein using $10 \mathrm{~mL}$ clotting tubes, centrifuged at 3,000 rpm for 30 minutes at $4^{\circ} \mathrm{C}$, aliquoted into separate Eppendorf tubes, immersed in liquid nitrogen, and then stored at $-80^{\circ} \mathrm{C}$. Written informed consent was obtained from all the participants, which was reviewed and approved by the Ethics Committee of the Third Affiliated Hospital of Wenzhou Medical University.

Table I Correlations between serum miR-503 expression levels and clinical parameters

\begin{tabular}{|c|c|c|c|}
\hline Variables & $\begin{array}{l}\text { GC patients } \\
(n=68)\end{array}$ & $\begin{array}{l}\text { Healthy controls } \\
(n=30)\end{array}$ & $P$-value \\
\hline Age (years) & & & 0.237 \\
\hline$<60$ & 25 & 16 & \\
\hline$\geq 60$ & 43 & 14 & \\
\hline Sex & & & 0.205 \\
\hline Male & 43 & 20 & \\
\hline Female & 25 & 10 & \\
\hline Tumor size $(\mathrm{cm})$ & & & 0.132 \\
\hline$<5$ & 40 & & \\
\hline$\geq 5$ & 28 & & \\
\hline Histological grade & & & 0.394 \\
\hline Well and moderate & 19 & & \\
\hline Poor and others & 49 & & \\
\hline Depth of tumor & & & 0.152 \\
\hline TI, T2 & 17 & & \\
\hline T3, T4 & 51 & & \\
\hline Lymphatic metastasis & & & $0.029 *$ \\
\hline Absent & 27 & & \\
\hline Present & 41 & & \\
\hline Distant metastasis & & & 0.036 \\
\hline Absent & 52 & & \\
\hline Present & 16 & & \\
\hline Tumor stage $\mathrm{e}^{\mathrm{a}}$ & & & $0.03 I^{*}$ \\
\hline I and II & 24 & & \\
\hline III and IV & 44 & & \\
\hline
\end{tabular}

Notes: according to the TNM criteria. $* P<0.05$.

Abbreviation: GC, gastric cancer. 


\section{Detection of serum CEA}

Serum carcinoembryonic antigen (CEA) levels in the sera of the GC patients and healthy individuals were determined at the Department of Clinical Laboratory of our hospital.

\section{Extraction of RNA and miRNA detection}

Total RNA of human tissue and serum samples were extracted using the BioTeKe miRNA extraction kit (BioTeKe Corporation, Beijing, People's Republic of China) according to the manufacturer's instructions. The expression levels of miR-503 in samples were measured by real-time reverse transcription quantitative polymerase chain reaction (RT-qPCR). Reverse transcription was performed using a TaqMan MicroRNA Reverse Transcription Kit (Applied Biosystems, Foster City, CA, USA). MiRNA was quantified on the CFX96 ${ }^{\mathrm{TM}}$ Real-Time PCR Detection System (Bio-Rad Laboratories, Hercules, CA, USA). The data were normalized against the U6 small nuclear RNA. All reactions were conducted in triplicate. The miR-503 expression level was calculated by cycle threshold $(\mathrm{Ct})$ values with SDS 2.0 software (Applied Biosystems). Relative miR-503 levels were calculated using the $2^{-\Delta \Delta \mathrm{Ct}}$ method.

\section{Statistical analysis}

SPSS v. 20.0 (SPSS Inc., Chicago, IL, USA) was used for the statistical analysis. Student's $t$-test and Mann-Whitney $U$-test were used to compare other group comparisons. Kaplan-Meier analysis with the log-rank test was performed for disease progression. The area under the receiver operating characteristic (ROC) curve was used to assess the feasibility of tissue and serum miR-503 as a diagnostic tool for GC. The MedCalc 10.4.7.0 (MedCalc, Mariakerke, Belgium) software was used to perform the ROC analysis. Spearman's correlation was used to evaluate the relationships between the relative expression levels of miR-503 and clinicopathological data and CEA in GC tissues and sera. $P$-value $<0.05$ was considered statistically significant.

\section{Results}

\section{MiR-503 is downregulated in GC tissues}

To investigate the expression level of miR-503 in human GC, we monitored its expression in 68 pairs of human GC and adjacent normal mucosa tissues (ANT). We found that miR-503 expression was decreased in GC tissues compared to the ANT ( $P=0.031$, Figure 1A). To further examine the role of miR-503 in human GC development, we divided all GC patients into two groups: in one group, tumors were excised from 54 patients with distant or lymph node metastasis, while in the other group, tumors were excised from 14 patients without metastasis. The RT-qPCR analysis results showed that the expression levels of miR-503 were significantly lower in GC patients with metastasis compared with those without metastasis $(P<0.001$, Figure 1B).

\section{Association of miR-503 expression with clinicopathological parameters in GC}

According to the results of RT-qPCR, we evaluated the association between miR-503 expression and the clinicopathological parameters of patients. As summarized in Table 1, miR-503 expression was significantly correlated with lymphatic metastasis $(P=0.029)$ and TNM stage $(P=0.031)$. However, no correlation was observed between the expression levels of miR-503 and other clinicopathological factors, including age, sex, lesion size, histology, and distant metastasis (all $P>0.05$, Table 1).
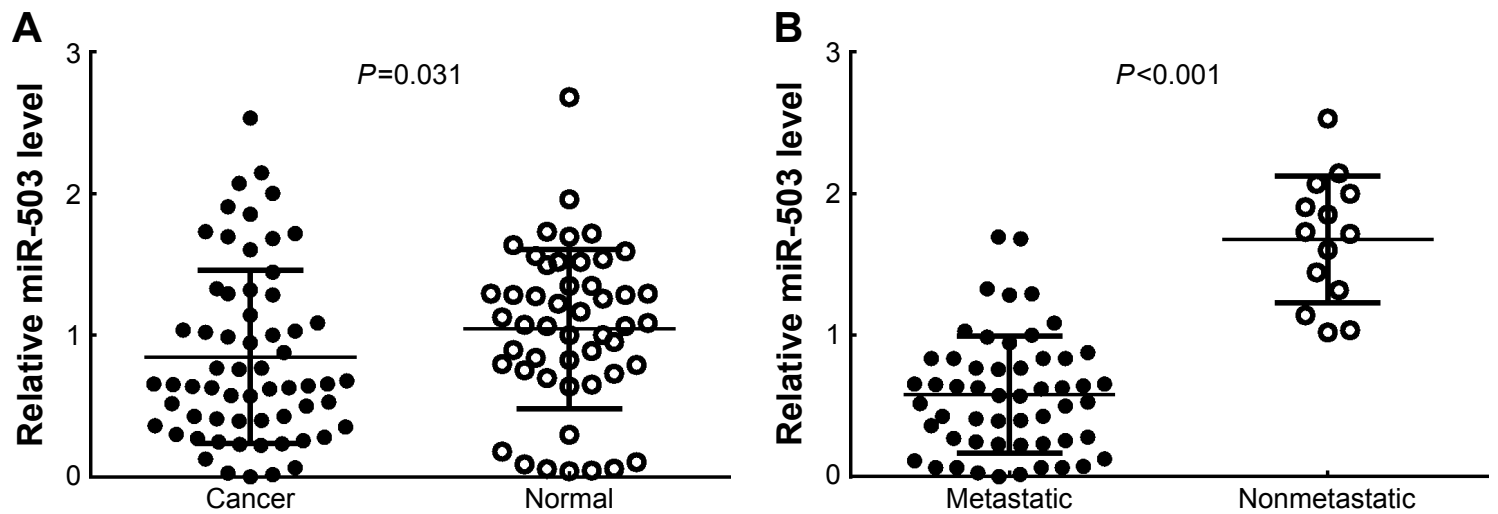

Figure I MiR-503 downregulation in GC tissues.

Notes: (A) The expression level of miR-503 in GC and adjacent normal mucosal tissues $(n=68)$ was determined by RT-qPCR analysis. (B) MiR-503 expression levels in metastatic $(n=54)$ and nonmetastatic $(n=14)$ GC.

Abbreviations: GC, gastric cancer; RT-qPCR, real-time reverse transcription quantitative polymerase chain reaction. 


\section{Diagnostic and prognostic significance of miR-503 in the tissues of GC patients}

The ability of miR-503 expression to discriminate between normal and tumor tissue samples was evaluated by ROC curve analyses. According to ROC curve, when the miR-503 expression $2^{-\Delta \Delta \mathrm{Ct}}$ value was 0.694 , miR-503 showed the best sensitivity and specificity with an AUC of $0.862(P=0.045$; 95\% confidence interval: 0.757-0.967) (Figure 2A), suggesting that miR-503 expression in GC samples can discriminate malignant tissues from normal tissues and may therefore be used as a diagnostic biomarker for GC. To determine whether the miR-503 expression levels in tumor tissues correlate with the prognosis of the GC patients, the patients were divided into low-miR-503 group $\left(2^{-\Delta \Delta C t}\right.$ value $\left.<0.694, n=42\right)$ and high-miR-503 group ( $2^{-\Delta \Delta C t}$ value $\geq 0.694, n=26$ ). The results of the Kaplan-Meier curves with log-rank analysis showed that the low-miR-503 group conferred a significantly worse OS (log rank $P=0.021$, Figure 2B).

\section{Expression levels and diagnostic significance of miR-503 in the sera of GC patients}

MiR-503 expression was determined in the sera of GC patients and healthy volunteers. As shown in Figure 3A, the expression level of miR-503 in the serum of $32 \mathrm{GC}$ patients was significantly lower than that of the healthy volunteers. Interestingly, when the relationship between the serum miR-503 level and traditional tumor marker CEA (Figure 3B) was analyzed, we found that the miR-503 level was inversely associated with CEA ( $r=-0.624, P<0.001$, Figure 3C). ROC analysis was performed to further detect the diagnostic value of miR-503 and CEA. Regarding the area under the ROC curve, miR-503 showed a higher value at $0.889(P=0.006)$, with a sensitivity of $96.8 \%$ and a specificity of $79.4 \%$, while the value for CEA was $0.681(P=0.048)$, with a sensitivity of $82.5 \%$ and a specificity of $53 \%$ (Figure 3D). Our findings suggested that the diagnostic value of miR-503 was more effective than CEA in discriminating GC patients from healthy individuals.

\section{Discussion}

In this study, we demonstrated that miR-503 expression was significantly downregulated in GC tissues. Furthermore, the expression level of miR-503 was significantly lower in patients with metastasis than patients without metastasis. Our ROC and Kaplan-Meier analysis results showed that miR-503 can be utilized as a diagnostic biomarker for GC and that low miR-503 levels conferred worse OS of GC patients. Our results also showed that the miR-503 level was different between GC patients and healthy participants, and its expression profiles can be used to differentiate GC patients from healthy people. In addition, the diagnostic performance of miR-503 was significantly higher than that of CEA. As a widely useful diagnostic biomarker for GC patients, CEA is a tumor biomarker used in the clinical diagnosis of GC, whereas its specificity and sensitivity are relatively poor. ${ }^{11}$ In addition to present a higher diagnostic value, the sensitivity and specificity of miR-503 were also
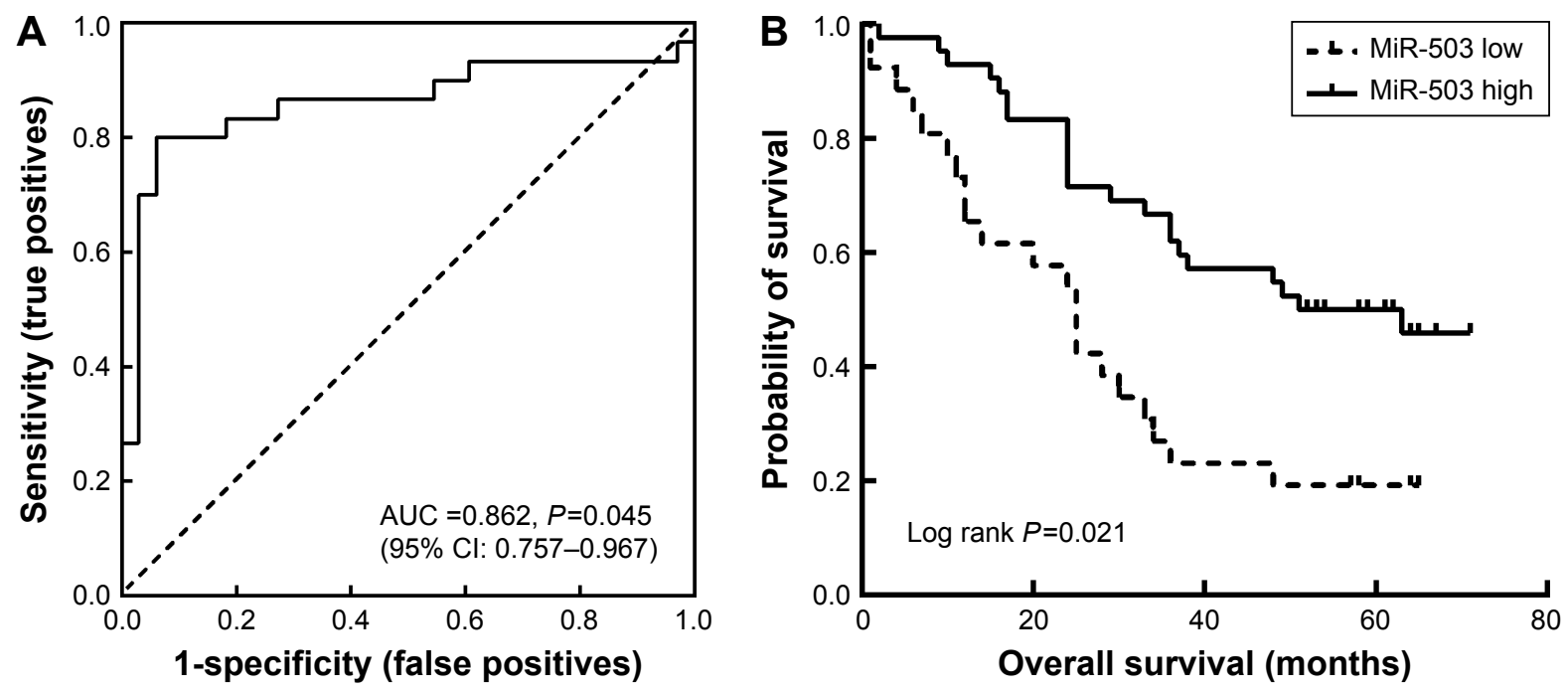

Figure 2 Diagnostic and prognostic significance of miR-503 in tissues of GC.

Notes: (A) ROC curve analysis showing performance of miR-503 expression to discriminate between malignant and nonmalignant tissue samples. (B) Kaplan-Meier analysis and log-rank test for overall survival of GC patients based on miR-503 expression.

Abbreviations: GC, gastric cancer; ROC, receiver operating characteristic; AUC, area under the curve; $\mathrm{Cl}$, confidence interval. 

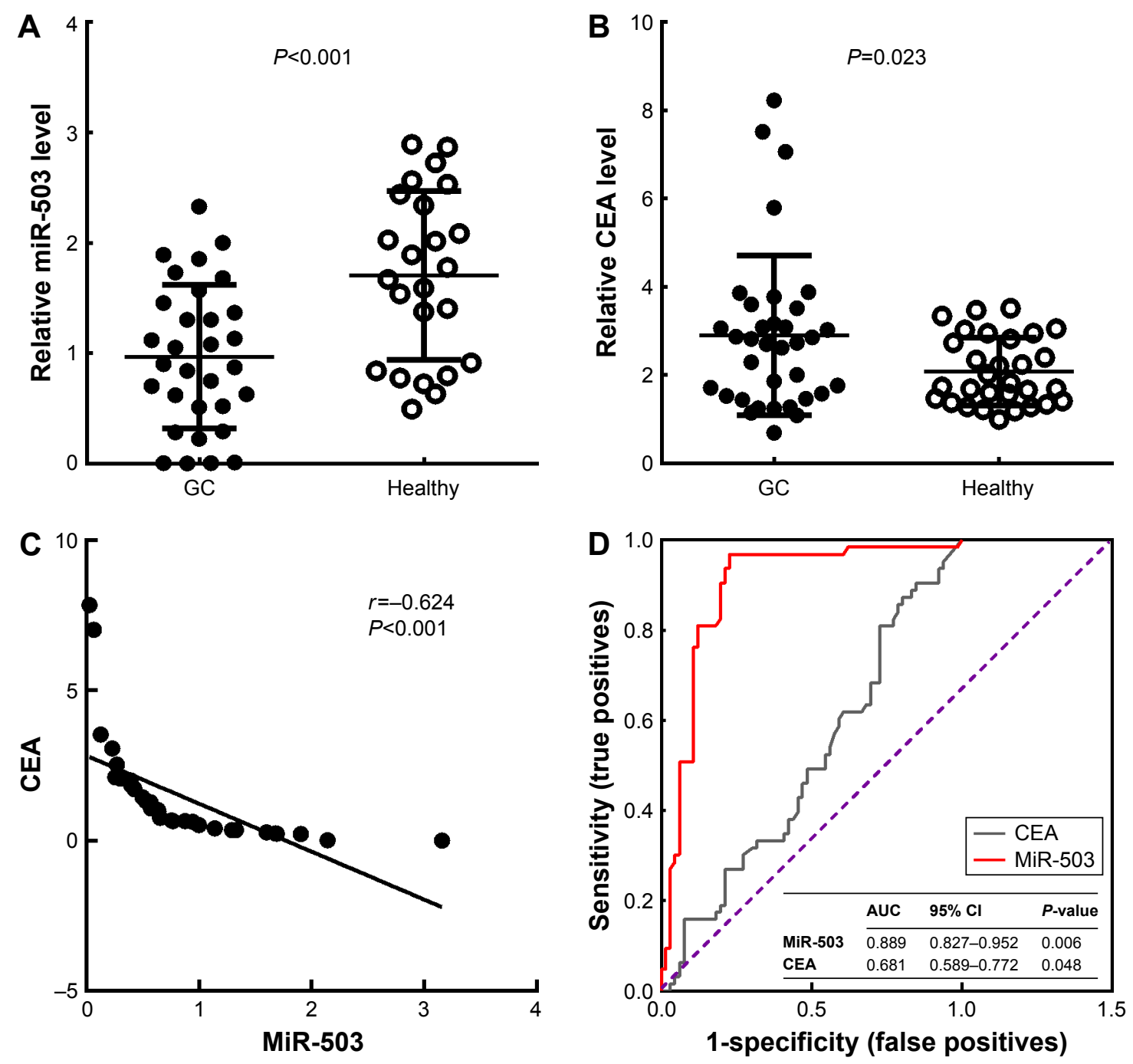

Figure 3 MiR-503 expression and diagnostic significance in serum of GC patients and healthy controls.

Notes: (A) The expression level of miR-503 in serum of GC patients $(n=32)$ or healthy participants $(n=32)$ was determined by RT-qPCR analysis. (B) The expression level of CEA in serum of GC patients ( $n=32$ ) or healthy participants $(n=32)$ was determined by RT-qPCR analysis. (C). Correlation of the expression of miR-503 and CEA in GC patients. (D) ROC analysis curve of CEA and miR-503 expression in the serum of the GC patients and healthy controls.

Abbreviations: GC, gastric cancer; RT-qPCR, real-time reverse transcription quantitative polymerase chain reaction; CEA, carcinoembryonic antigen; ROC, receiver operating characteristic; $\mathrm{AUC}$, area under the curve; $\mathrm{Cl}$, confidence interval.

higher than that of CEA. Our current data indicate that serum miR-503 should be further developed as a novel prognostic biomarker and powerful tool for the early diagnosis of GC.

Studies have revealed that miRNAs constitute a robust regulatory network by functioning as posttranscriptional regulators. ${ }^{12}$ Dysregulation of miRNAs has been reported in various human malignancies, ${ }^{13-15}$ and multiple deregulated miRNAs have been implicated in biological and pathological process of GC. ${ }^{16-20}$ Based on the deregulated miRNAs' expression profiling and their association with the intrinsic characteristics of GC, specific miRNAs could be utilized as new noninvasive prognostic and diagnostic biomarkers for cancers. ${ }^{21,22}$ In addition to cancer tissues, expression profiles of miRNAs are also altered in peripheral blood of patients with GCs. ${ }^{23-26}$ If GC is diagnosed at an early stage, there is a good chance of a cure using advanced endoscopic resection techniques (eg, endoscopic mucosal resection and endoscopic submucosal dissection) or other less invasively surgical regimens. Unfortunately, although currently there are some new tumor markers such as CA72-4, there is still no appropriate screening strategy available for the screening, diagnosis, or postsurgical surveillance of GC. The properties of miRNAs provide a huge potential for establishing noninvasive and reliable GC-screening biomarkers. In this study, we focused on the functional miR-503, which was reported to deregulate in GC tissues, and examined its expression profiling in tissue and serum samples obtained from GC patients and healthy individuals by RT-qPCR. We found that the miR-503 levels 
were lower in GC patients than in ANT of healthy individuals. Therefore, the investigation of miR-503 levels in tissue and serum of GC patients, as present in this study, may be of great clinical interest.

As shown by previous studies, miR-503 can function as a tumor suppressor, which is well reflected in the present study that showed lower expression of miR-503 was strongly correlated with higher tumor burden of GC. ${ }^{8,9}$ Moreover, our results showed negative correlations between miR-503 expression and serum CEA level, which may be to some extent a good explanation for the potential mechanism of miR-503 in GC. Functional assays demonstrated that miR-503 suppresses EMT in GC cells by downregulating fibronectin, vimentin, N-cadherin, and SNAIL protein levels. ${ }^{8}$ Similarly, miR-503 was reported to inhibit the growth and invasiveness of prostate cancer, hepatocellular carcinoma, and breast cancer by downregulating the expression of RNF31, ${ }^{27}$ PRMT1, ${ }^{28}$ and CCND1, ${ }^{29}$ respectively. In addition, overexpression of miR-503 sensitized human GC cell lines to cisplatin by targeting IGF1R and BCL2. ${ }^{9}$ All these together suggest that miR-503 may have different targets and perform specific functions in different physiological and pathological processes of different malignancies, thereby exhibiting different expression profiles in specific tumor types and stages. Therefore, it is understandable that miR-503 expression was significantly lower in patients with metastasis.

The current findings may have some limitations. 1) Our results show that miR-503 expression was downregulated in a small cohort of GC patients; the results require confirmation in further large-scale and multicentral studies before moving discovery into clinical practice. 2) The expression of miR503 was downregulated in GC, and the cutoff points that can be detected in clinical samples for downexpressed miRNAs were very low, which may be a main obstacle for its clinical translation. Besides, since the function of miR-503 in GC pathogenesis has been shown thoroughly, further studies are needed to explain the underlying pathogenetic mechanism of this miRNA, which will undoubtedly be helpful for future personalized therapy.

In conclusion, the present study showed that miR-503 expression was downregulated in GC and was associated with the prognosis of GC. Moreover, there were negative correlations between miR-503 expression and malignant phenotypes of GC, as well as the serum expression level of CEA in GC patients. Furthermore, our results showed that miR-503 may be used as a biomarker for GC diagnosis and postsurgical surveillance. An increased number of future indepth studies are required to confirm the diagnostic values of
miR-503 in diagnosis and prognosis of GC. Further studies on the antitumor mechanisms of miR-503 may contribute to the development of new effective approaches for GC therapy.

\section{Disclosure}

The authors report no conflicts of interest in this work.

\section{References}

1. Torre LA, Bray F, Siegel RL, Ferlay J, Lortet-Tieulent J, Jemal A. Global cancer statistics, 2012. CA Cancer J Clin. 2015;65(2):87-108.

2. Leja M, You W, Camargo MC, Saito H. Implementation of gastric cancer screening - the global experience. Best Pract Res Clin Gastroenterol. 2014;28(6):1093-1106.

3. Rutnam ZJ, Yang BB. The involvement of microRNAs in malignant transformation. Histol Histopathol. 2012;27(10):1263-1270.

4. Winter J, Jung S, Keller S, Gregory RI, Diederichs S. Many roads to maturity: microRNA biogenesis pathways and their regulation. Nat Cell Biol. 2009;11(3):228-234.

5. Skrzypski M, Dziadziuszko R, Jassem J. MicroRNA in lung cancer diagnostics and treatment. Mutat Res. 2011;717(1-2):25-31.

6. Mitchell PS, Parkin RK, Kroh EM, et al. Circulating microRNAs as stable blood-based markers for cancer detection. Proc Natl Acad Sci US A. 2008;105(30):10513-10518.

7. Fang Y, Yao Q, Chen Z, et al. Genetic and molecular alterations in pancreatic cancer: implications for personalized medicine. Med Sci Monit. 2013;19:916-926.

8. Peng Y, Liu YM, Li LC, Wang LL, Wu XL. microRNA-503 inhibits gastric cancer cell growth and epithelial-to-mesenchymal transition. Oncol Lett. 2014; 7(4):1233-1238.

9. Wang T, Ge G, Ding Y, et al. MiR-503 regulates cisplatin resistance of human gastric cancer cell lines by targeting IGF1R and BCL2. Chin Med J (Engl). 2014;127(12):2357-2362.

10. Lauwers GY, Carneiro F, Graham DY, Curado MP. Tumours of the stomach. In: WHO Classification of Tumours of the Digestive System, editors: Bosman FT, Carneiro F, Hruban RH, Theise ND, 4th Edition. Lyon: IARC; 2010:48-59.

11. Liu X, Qiu H, Liu J, et al. Combined preoperative concentrations of CEA, CA 19-9, and 72-4 for predicting outcomes in patients with gastric cancer after curative resection. Oncotarget. Epub 2016 Apr 27.

12. Bartel DP. MicroRNAs: genomics, biogenesis, mechanism, and function. Cell. 2004;116(2):281-297.

13. Leidinger P, Keller A, Borries A, et al. High-throughput miRNA profiling of human melanoma blood samples. BMC Cancer. 2010;10:262.

14. Huang Z, Huang D, Ni S, Peng Z, Sheng W, Du X. Plasma microRNAs are promising novel biomarkers for early detection of colorectal cancer. Int J Cancer J. 2010;127(1):118-126.

15. Mueller DW, Bosserhoff AK. Role of miRNAs in the progression of malignant melanoma. Br J Cancer. 2009;101(4):551-556.

16. Li Z, Zhang G, Li D, et al. Methylation-associated silencing of miR495 inhibit the migration and invasion of human gastric cancer cells by directly targeting PRL-3. Biochem Biophys Res Commun. 2015; 456(1):344-350.

17. An Y, Zhang Z, Shang Y, et al. miR-23b-3p regulates the chemoresistance of gastric cancer cells by targeting ATG12 and HMGB2. Cell Death Dis. 2015;6:e1766.

18. Li F, Liu B, Gao Y, et al. Upregulation of microRNA-107 induces proliferation in human gastric cancer cells by targeting the transcription factor FOXO1. FEBS Lett. 2014;588(4):538-544.

19. Duan JH, Fang L. MicroRNA-92 promotes gastric cancer cell proliferation and invasion through targeting FXR. Tumour Biol. 2014;35(11): 11013-11019.

20. Feng L, Xie Y, Zhang H, Wu Y. miR-107 targets cyclin-dependent kinase 6 expression, induces cell cycle G1 arrest and inhibits invasion in gastric cancer cells. Med Oncol. 2012;29(2):856-863. 
21. Wang M, Zhao C, Shi H, et al. Deregulated microRNAs in gastric cancer tissue-derived mesenchymal stem cells: novel biomarkers and a mechanism for gastric cancer. Br J Cancer. 2014;110(5):1199-1210.

22. Kim CH, Kim HK, Rettig RL, et al. miRNA signature associated with outcome of gastric cancer patients following chemotherapy. BMC Med Genomics. 2011;4:79.

23. Imaoka H, Toiyama Y, Okigami M, et al. Circulating microRNA-203 predicts metastases, early recurrence, and poor prognosis in human gastric cancer. Gastric Cancer. 2016;19(3):744-753.

24. Ayremlou N, Mozdarani H, Mowla SJ, Delavari A. Increased levels of serum and tissue miR-107 in human gastric cancer: Correlation with tumor hypoxia. Cancer Biomark. 2015;15(6):851-860.

25. Zeng Q, Jin C, Chen W, et al. Downregulation of serum miR-17 and miR-106b levels in gastric cancer and benign gastric diseases. Chin J Cancer Res. 2014;26(6):711-716.
26. Wang $\mathrm{R}$, Wen $\mathrm{H}, \mathrm{Xu} \mathrm{Y}$, et al. Circulating microRNAs as a novel class of diagnostic biomarkers in gastrointestinal tumors detection: a metaanalysis based on 42 articles. PloS One. 2014;9(11):e113401.

27. Guo J, Liu X, Wang M. miR-503 suppresses tumor cell proliferation and metastasis by directly targeting RNF31 in prostate cancer. Biochem Biophys Res Commun. 2015;464(4):1302-1308.

28. Li B, Liu L, Li X, Wu L. miR-503 suppresses metastasis of hepatocellular carcinoma cell by targeting PRMT1. Biochem Biophys Res Commun. 2015;464(4):982-987.

29. Long J, Ou C, Xia H, Zhu Y, Liu D. MiR-503 inhibited cell proliferation of human breast cancer cells by suppressing CCND1 expression. Tumour Biol. 2015;36(11):8697-8702.

\section{Publish your work in this journal}

OncoTargets and Therapy is an international, peer-reviewed, open access journal focusing on the pathological basis of all cancers, potential targets for therapy and treatment protocols employed to improve the management of cancer patients. The journal also focuses on the impact of management programs and new therapeutic agents and protocols on

\section{Dovepress}

patient perspectives such as quality of life, adherence and satisfaction. The manuscript management system is completely online and includes a very quick and fair peer-review system, which is all easy to use. Visit http://www.dovepress.com/testimonials.php to read real quotes from published authors.

Submit your manuscript here: http://www.dovepress.com/oncotargets-and-therapy-journal 